\title{
Influence of Functionalization Degree on the Rheological Properties of Isocyanate-Functionalized Chitin- and Chitosan-Based Chemical Oleogels for Lubricant Applications
}

\author{
Rocío Gallego ${ }^{1}$, Mercedes González ${ }^{1}$, Jesús F. Arteaga ${ }^{1,2, *}$, Concepción Valencia ${ }^{1,3}$ and \\ José M. Franco ${ }^{1,3}$
}

1 Department of Chemical Engineering, Physical Chemistry and Organic Chemistry, University of Huelva, Campus El Carmen, Campus ceiA3, 21071 Huelva, Spain;

E-Mails: rocio.gallego@diq.uhu.es (R.G.); mercedes.gonzalez@diq.uhu.es (M.G.); barragan@uhu.es (C.V.); franco@uhu.es (J.M.F.)

2 CIQSO - Center for Research in Sustainable Chemistry, University of Huelva, 21071 Huelva, Spain

3 Pro $^{2}$ TecS - Chemical Product and Process Technology Center, University of Huelva, 21071 Huelva, Spain

* Author to whom correspondence should be addressed; E-Mail: jesus.fernandez@diq.uhu.es; Tel.: +34-959-219-999; Fax: +34-959-219-983.

Received: 30 May 2014; in revised form: 27 June 2014 / Accepted: 28 June 2014 /

Published: 8 July 2014

\begin{abstract}
This work deals with the influence of functionalization degree on the thermogravimetric and rheological behaviour of NCO-functionalized chitosan- and chitin-based oleogels. Chitosan and chitin were functionalized using different proportions of 1,6-hexamethylene diisocyanate (HMDI) and subsequently dispersed in castor oil to promote the chemical reaction between the $-\mathrm{NCO}$ group of the modified biopolymer and the $-\mathrm{OH}$ group located in the ricinoleic fatty acid chain of castor oil, thus resulting in different oleogels with specific thermogravimetric and rheological characteristics. Biopolymers and oleogels were characterized through Fourier transform infrared spectroscopy (FTIR) and thermogravimetric analysis (TGA). Small-amplitude oscillatory shear (SAOS) measurements were performed on the oleogels. Oleogels presented suitable thermal resistance, despite the fact that the inclusion of HMDI moieties in the polymer structure led to a reduction in the onset temperature of thermal degradation. The insertion of low amounts of HMDI in both chitin and chitosan produces a drastic reduction in the values of oleogel viscoelastic functions but, above a critical threshold, they increase with
\end{abstract}


the functionalization degree so that isocyanate functionalization results in a chemical tool to modulate oleogel rheological response. Several NCO-functionalized chitosan- and chitin-based oleogel formulations present suitable thermal resistance and rheological characteristics to be proposed as bio-based alternatives to traditional lubricating greases.

Keywords: biopolymer; isocyanate-functionalized chitin and chitosan; oleogel; lubricating greases; rheology

\section{Introduction}

Organogels are generating great interest and expectation in a wide spectrum of applications such as medical and pharmaceutical [1-4], development of new materials with specific optical and mechanical properties [5-7], matrixes for food systems [8,9], art conservation [10,11], etc. The term organogel refers to a variety of soft materials, from viscoelastic liquid-like to solid-like, comprised of an organic liquid and some molecules, i.e., organogelators, which are able to produce self-assembling and structure the organic medium [12]. In most cases, low-molecular weight amphiphilic molecules which impart thermo-reversible properties are responsible for the gelling behaviour [13-17]. However, some biomacromolecules like natural waxes, resins and biopolymers with gelling properties in oily media are gaining special attention [18-26]. Among these studies, lignocellulosic materials and cellulose derivatives have been used to obtain gel-like dispersions in vegetable oils, which may be potentially applied as environmentally friendly lubricating grease formulations [27-32].

In the frame of the general tendency to promote the replacement of non-renewable raw materials by renewable resources in order to avoid the impact that process technologies and products of different industrial sectors cause on the environment, the lubricant industry is especially involved [33,34]. In this sense, different alternatives based on modifications of vegetable oils have been proposed to replace mineral and synthetic lubricant base oils [35-40]. Although vegetable oils are being increasingly used as bio-based lubes, the substitution of traditional thickening agents, such as metallic soaps and polyureas, in lubricating greases by others derived from renewable resources is a much more complicated task. For instance, some limitations regarding physical and mechanical stabilities and thermo-rheological behaviour of previously mentioned cellulosic derivatives-based gel-like dispersions [27-29] were pointed out as a consequence of a certain degree of chemical incompatibility between oil and such polar biopolymers. Most of these problems can be overcome by inducing the formation of chemical gels, for instance by dispersing reactive isocyanate-functionalized methylcellulose in the vegetable oil medium [41,42]. Following the same procedure and based on some preliminary studies [43], the possibility of using NCO-functionalized chitin and chitosan to chemically gel castor oil is explored in this work. As well known, chitin is one of the most abundant organic materials, being second only to cellulose in the amount produced annually by biosynthesis. It is an important constituent of the exoskeleton in animals especially in crustaceans, molluscs and insects and the principal fibrillar polymer in the cell wall of certain fungi $[44,45]$. Chitosan is typically obtained by deacetylation of chitin under alkaline conditions in a variety of useful forms, characterized by displaying interesting properties such as biocompatibility or biodegradability [46-48], which also 
produces non-toxic, non-immunogenic and non-carcinogenic degradation products [49,50]. Nowadays, there is a growing interest in the chemical modification of chitosan in order to improve its solubility or the cross-linking ability in different media and therefore widen its applications [51-54]. The reaction between chitosan and diisocyanate compounds has been previously promoted to produce bio-polyurethanes for the development of molecular carriers in the biomedical and environmental fields [55], beads for cholesterol absorption [56] or materials with controlled thermal or elastomeric properties [57,58]. As a continuation of the above mentioned research on the development of oleogels for lubricant purposes and taking into account the interest of these biopolymers, the effect of NCO-functionalization degree of chitin and chitosan on the rheological properties and thermal degradation of resulting chemical oleogels has been studied in this work.

\section{Experimental Section}

\subsection{Materials}

Castor oil (kinematic viscosity: $211 \mathrm{~mm}^{2} \cdot \mathrm{s}^{-1}$ at $40{ }^{\circ} \mathrm{C}$, Guinama, Spain) was selected as lubricant base oil to formulate the oleogel samples studied. Chitosan (degree of deacetylation: $86.3 \%$; viscosity average molecular weight: $2.3 \times 10^{5} \mathrm{~g} \cdot \mathrm{mol}^{-1}$ ) and chitin (degree of deacetylation: $7.3 \%$; viscosity average molecular weight: $5.4 \times 10^{5} \mathrm{~g} \cdot \mathrm{mol}^{-1}$ ), purchased from Fraken (Qingdao, China), were modified with 1,6-hexamethylene diisocyanate (HMDI, purum grade, $\geq 98.0 \%$ ), supplied by Sigma-Aldrich (St. Louis, MO, USA). All other common reagents and solvents employed were purchased from Sigma-Aldrich.

\subsection{General Methods}

Functionalization reactions were performed in flasks that were previously flame-dried under a positive flow of argon to eliminate surface moisture. HMDI was stored at $4{ }^{\circ} \mathrm{C}$ and handled under argon atmosphere. Solvents were purified according to standard literature techniques and stored under argon. Toluene was freshly distilled immediately prior to use from sodium/benzophenone and strictly deoxygenated for $30 \mathrm{~min}$ under argon. Reagents were purchased at the higher commercial quality and used without further purification, unless otherwise stated. Characterization tests were performed at least in duplicate.

\subsection{Functionalization Reaction of Chitosan and Chitin with HMDI}

The functionalization reactions of chitosan and chitin with HMDI were carried out according to the protocol described in preliminary studies [43]. Chitosan or chitin was added to a bottom round flask with toluene while stirring at room temperature until it became a suspension. Then, triethylamine $\left(\mathrm{Et}_{3} \mathrm{~N}\right)$ and HMDI were also added to the system, the last one drop-wisely. The solution was vigorously stirred at room temperature for $24 \mathrm{~h}$. Afterwards, the mixture was vacuum filtered and the resulting product was immediately used to prepare the oleogels. The amount of HMDI, Et ${ }_{3} \mathrm{~N}$ and toluene was modified with the aim of achieving different functionalization degrees of $-\mathrm{OH}$ and $-\mathrm{NH}_{2}$ groups in the chitosan and chitin derivatives synthesized. The different molar ratios of $\mathrm{HMDI}_{2} \mathrm{Et}_{3} \mathrm{~N}$ 
and toluene with respect to free $-\mathrm{OH}$ and $-\mathrm{NH}_{2}$ groups in the non-functionalized chitin and chitosan monomers are listed in Table 1.

Table 1. Molar ratios of chitin or chitosan, 1,6-hexamethylene diisocyanate (HMDI), triethylamine $\left(\mathrm{Et}_{3} \mathrm{~N}\right)$ and toluene used in each functionalization reaction.

\begin{tabular}{ccccc}
\hline $\begin{array}{c}\text { Functionalized } \\
\text { Polymer }^{\text {\& }}\end{array}$ & $\begin{array}{c}\text { Starting material } \\
\text { [equiv.] * }\end{array}$ & $\begin{array}{c}\text { HMDI } \\
\text { [equiv.] * }\end{array}$ & $\begin{array}{c}\mathbf{E t}_{3} \mathbf{N} \\
\text { [equiv.] * }\end{array}$ & $\begin{array}{c}\text { Toluene } \\
\text { [mL] }\end{array}$ \\
\hline CSAN-1 & 1.00 (Chitosan) & 0.50 & 1.00 & 100 \\
CSAN-2 & 1.00 (Chitosan) & 0.25 & 0.50 & 100 \\
CSAN-3 & 1.00 (Chitosan) & 0.13 & 0.25 & 100 \\
CSAN-4 & 1.00 (Chitosan) & 0.06 & 0.13 & 100 \\
CTIN-1 & 1.00 (Chitin) & 0.50 & 1.0 & 100 \\
CTIN-2 & 1.00 (Chitin) & 0.25 & 0.50 & 100 \\
CTIN-3 & 1.00 (Chitin) & 0.13 & 0.25 & 100 \\
CTIN-4 & 1.00 (Chitin) & 0.06 & 0.13 & 100 \\
\hline
\end{tabular}

\& The acronym "CSAN" corresponds to chitosan polymers and "CTIN" to chitin polymers; the adjacent number (1-4) define a different degree of functionalization for each type of polymer. * Molar ratio between moles of free $-\mathrm{OH}$ and $-\mathrm{NH}_{2}$ groups present in each monomer of starting chitosan and chitin, and moles of each of the reagents.

\subsection{Preparation of Oleogels}

Oleogel samples were prepared in an open vessel (batches of $60 \mathrm{~g}$ ), using a controlled-rotational speed mixing device (70 rpm) RW 20 (Ika, Staufen, Germany), equipped with an anchor impeller to disperse the different biopolymers in the oil. Functionalized biopolymers were slowly added to the castor oil at different concentrations (see Table 2). The mixing process was maintained at $70 \mathrm{rpm}$ for $24 \mathrm{~h}$ at room temperature. Finally, the resulting dispersion was homogenized with an Ultra-Turrax T50 (Ika) rotor-stator turbine, at $8800 \mathrm{rpm}$ for $1 \mathrm{~min}$. Table 2 also shows the codes applied for each oleogel sample.

\subsection{Thermogravimetric Analysis (TGA)}

Measurements of mass loss versus temperature were carried out by using a Thermogravimetric analyser, model Q-50 (TA Instruments, New Castle, DE, USA) under $\mathrm{N}_{2}$ purge. Typically, 5-10 mg of sample were placed on a platinum pan and heated from $30^{\circ} \mathrm{C}$ to $600{ }^{\circ} \mathrm{C}$, at $10^{\circ} \mathrm{C} \cdot \mathrm{min}^{-1}$.

\subsection{Fourier Transform Infrared (FTIR) Spectroscopy}

FTIR spectra were obtained with a Digilab FTS3500ARX (Varian, Palo Alto, CA, USA) apparatus. Chitin and chitosan samples were prepared as $\mathrm{KBr}$ pellets, whereas a small drop of oleogels was placed between two $\mathrm{KBr}$ disks $(32 \mathrm{~mm} \times 3 \mathrm{~mm})$. In both cases, the set was placed into an appropriate sample holder. The spectra were obtained in a wavenumber range of $400-4000 \mathrm{~cm}^{-1}$, at $4 \mathrm{~cm}^{-1}$ resolution, in the transmission mode. The degree of deacetylation provided in Section 2.1 for original 
chitin and chitosan samples were determined by FTIR according to the method described by Baxter et al. [59].

Table 2. Composition of oleogel formulations studied.

\begin{tabular}{ccc}
\hline $\begin{array}{c}\text { Thickener } \\
\text { agent }\end{array}$ & $\begin{array}{c}\text { Concentration } \\
{[\mathbf{\%}, \mathbf{w} / \mathbf{w}]}\end{array}$ & $\begin{array}{c}\text { Code } \\
\text { Applied * }\end{array}$ \\
\hline Chitosan & 30 & CSAN-30 \\
CSAN-1 & 30 & CSAN-1-30 \\
CSAN-2 & 30 & CSAN-2-30 \\
CSAN-3 & 30 & CSAN-3-30 \\
CSAN-4 & 30 & CSAN-4-30 \\
CSAN-3 & 15 & CSAN-3-15 \\
CSAN-3 & 25 & CSAN-3-25 \\
CSAN-3 & 35 & CSAN-3-35 \\
Chitin & 30 & CTIN-30 \\
CTIN-1 & 30 & CTIN-1-30 \\
CTIN-2 & 30 & CTIN-2-30 \\
CTIN-3 & 30 & CTIN-3-30 \\
CTIN-4 & 30 & CTIN-4-30 \\
CTIN-2 & 15 & CTIN-2-15 \\
CTIN-2 & 25 & CTIN-2-25 \\
\hline
\end{tabular}

* Code Applied: polymer type (CTIN or CSAN)—different functionalization degree-concentration of the functionalized biopolymer in the oleogel prepared

\subsection{Rheological Characterization}

Rheological characterization of oleogels was carried out using controlled-stress rheometers (Gemini, Bohlin, Malvern Instruments Ltd., Worcestershire, United Kingdom, and Rheoscope, ThermoHaake, Karlsruhe, Germany). Small amplitude oscillatory shear (SAOS) tests were performed inside the linear viscoelastic region, using roughened plate-plate geometries $(20 \mathrm{~mm}, 25 \mathrm{~mm}$ and $35 \mathrm{~mm}$, and $1 \mathrm{~mm}$ gap), in a frequency range of $0.03-100 \mathrm{rad} \cdot \mathrm{s}^{-1}$, at $25^{\circ} \mathrm{C}$. Measurements were done one day, one week, one month and four months after oleogel preparation. Average molecular weight of original chitin and chitosan samples were determined by means of viscosity measurements according to the methods and Mark-Houwink-Sakurada equation constants provided by Poirier and Charlet [60] and Takahashi et al. [61], respectively.

\section{Results and Discussion}

\subsection{Spectroscopic Characterization}

Chemical modification of chitin and chitosan after functionalization with different proportions of HMDI and the occurrence of further reaction with $-\mathrm{OH}$ groups of castor oil were evaluated by FTIR. Figure 1 shows the FTIR spectra for non-functionalized chitin (Figure 1a) and chitosan (Figure 1d), selected NCO-functionalized samples, CTIN-1 (Figure 1b) and CSAN-1 (Figure 1e), and the corresponding oleogels prepared with these biopolymers and castor oil, CTIN-1-25 (Figure 1c) and 
CSAN-1-25 (Figure 1f). FTIR spectra of functionalized biopolymers were in concordance with the proposed chemical structure previously reported [43]. Table 3 lists the most relevant peaks detected in NCO-functionalized chitosan and chitin spectra. The reaction of chitin and chitosan with the $-\mathrm{NCO}$ groups of HMDI to form urethane linkages produces a significant reduction in the intensity of the band around $3200-3550 \mathrm{~cm}^{-1}$, corresponding to the $-\mathrm{OH}$ group in the molecules. The higher HMDI content, the more significant the reduction in this band is. The urethane bands (Figure $1 \mathrm{~b}, \mathrm{e}$ ) can be detected at approximately $3400 \mathrm{~cm}^{-1}$ and $1740 \mathrm{~cm}^{-1}$ for $\mathrm{N}-\mathrm{H}$ and $\mathrm{C}=\mathrm{O}$ stretching vibrations in the urethane linkage, respectively, and $1580 \mathrm{~cm}^{-1}$ for the $\mathrm{N}-\mathrm{H}$ bending vibration. Moreover, an intense peak at around $2270 \mathrm{~cm}^{-1}$ is noticed, confirming the presence of unreacted -NCO groups, as intended.

Figure 1. FTIR spectra for (a) chitin, (b) polymer CTIN-1; (c) oleogel CTIN-1-25;

(d) chitosan; (e) polymer CSAN-1 and (f) oleogel CSAN-1-25.

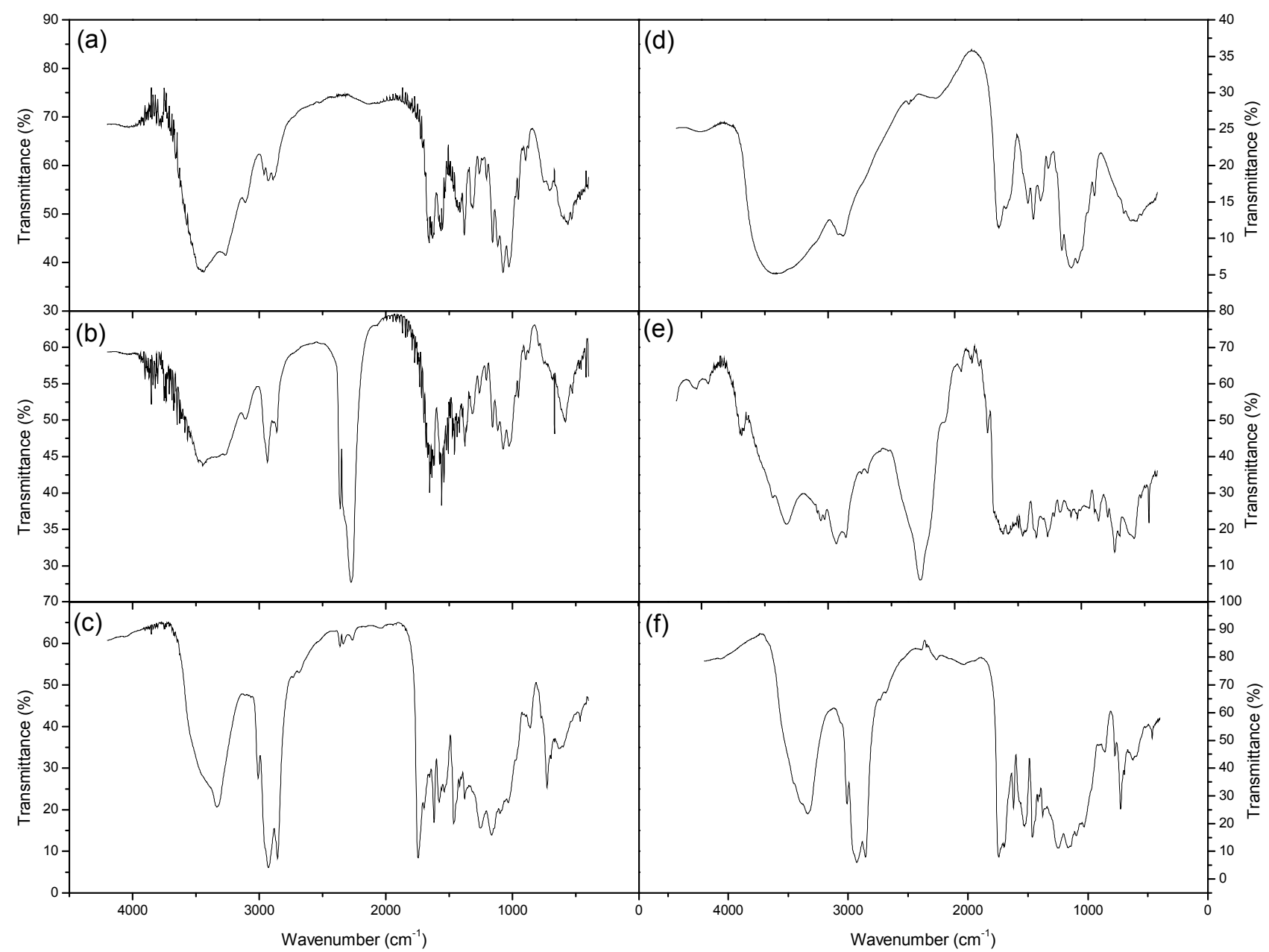

The functionalization degree was controlled by means of carrying out the reaction at room temperature, the amount of HMDI and $\mathrm{Et}_{3} \mathrm{~N}$ used and the reaction time ( $24 \mathrm{~h}$ in this case). Under these conditions, both a rapid attack of free - $\mathrm{OH}$ to -NCO groups of HMDI and an excessive cross-linking of -NCO groups present either in the unreacted HMDI or in the HMDI segments attached to the chitin or chitosan chains are significantly dampened. Thereby, the reaction can be controlled and allows obtaining selectively polymers with different degrees of functionalization. Finally, the reaction between the $-\mathrm{NCO}$ group of functionalized biopolymers and the $-\mathrm{OH}$ group of castor oil was also 
monitored by analyzing the FTIR spectra of oleogels (Figure 1c,f). As can be observed, the absorption band assigned to free -NCO groups at around $2271 \mathrm{~cm}^{-1}$ is negligible in the FTIR spectra of the oleogels, corroborating the occurrence of the reaction promoted.

Table 3. FTIR representative signals for selected NCO-functionalized chitin (CTIN-1) and chitosan (CSAN-1) samples.

\begin{tabular}{ccc}
\hline \multirow{2}{*}{ Functional group } & \multicolumn{2}{c}{ Signals } \\
\cline { 2 - 3 } & CTIN-1 $\left(\mathbf{c m}^{-\mathbf{1}}\right)$ & CSAN-1 $\left(\mathbf{c m}^{-\mathbf{1}}\right)$ \\
\hline$-\mathrm{OH}+=\mathrm{NH}$ & 3338 & 3330 \\
$\mathrm{C}-\mathrm{H}$ aliph & 2934 & 2936 \\
$\mathrm{~N}=\mathrm{C}=\mathrm{O}$ & 2275 & 2271 \\
$\mathrm{C}=\mathrm{O}$ & 1623 & 1741 \\
$\mathrm{~N}-\mathrm{H}$ & 1574 & 1580 \\
$\mathrm{C}-\mathrm{N}$ or $\mathrm{C}-\mathrm{OH}$ & 1259 & 1266 \\
$\mathrm{C}-\mathrm{O}$ or $\mathrm{C}-\mathrm{OC}$ & 1076 & 1082 \\
\hline
\end{tabular}

\subsection{Thermogravimetric Characterization}

Thermal degradation of chitin, chitosan and derived NCO-functionalized compounds was studied by means of thermogravimetric analysis (TGA). Figure 2 shows TGA curves, in the form of weight loss derivative function versus temperature, for chitosan (Figure 2a) and chitin (Figure 2b) functionalized in different degrees, compared to those obtained with the corresponding starting materials, i.e., non-functionalized biopolymers and HMDI. The onset temperature for thermally-induced weight loss $\left(T_{\text {onset }}\right)$, the maximum weight-loss rate temperature $\left(T_{\max }\right)$, the weight loss at the end of each decomposition step $(\Delta W)$ and the percentage of non-degraded residue were calculated from the thermogram of each sample studied (Table 4). Disregarding the initial weight loss between $40{ }^{\circ} \mathrm{C}$ and $80{ }^{\circ} \mathrm{C}$, which is inferior to $8 \%$, as a consequence of water evaporation, thermal degradation of non-functionalized chitosan and chitin takes place in one main stage. This main thermal event occurs in the range of $343-405{ }^{\circ} \mathrm{C}$ for chitin (Figure $2 \mathrm{~b}$ ) and is due to the degradation of the saccharide structure of the molecule, including the dehydration of saccharide rings and decomposition of the acetylated and deacetylated units [62]. In the case of chitosan (Figure 2a), thermal degradation starts at lower temperature, indicating poorer thermal stability, but in a wider range $\left(281-472{ }^{\circ} \mathrm{C}\right)$. This thermal range corresponds to the degradation peak of the part of the molecule that is deacetylated, showing a $T_{\max }$ at $301{ }^{\circ} \mathrm{C}$, and an overlapped small signal at around $390{ }^{\circ} \mathrm{C}$, due to the degradation of the acetylated part of the molecule.

On the other hand, for NCO-functionalized biopolymers, the inclusion of HMDI segments in the polymer structure reduces their thermal stabilities, with marked impact in the highest functionalized polymer, as expected (Figure 2, Table 4). Thermal degradation curves of NCO-functionalized chitosans exhibit two main events. The first one at around $83-179{ }^{\circ} \mathrm{C}$, with $T_{\max }$ at $116-153{ }^{\circ} \mathrm{C}$, corresponds to the loss of $-\mathrm{NCO}$ segments, which is corroborated with HMDI thermal degradation occurring at $137{ }^{\circ} \mathrm{C}$. As can be observed in Table 4, the $T_{\text {onset }}$ and, logically, weight loss for this degradation stage increases with - NCO content. The second main degradation stage, as previously discussed for the non-functionalized sample, is related to the loss of the deacetylated part of the 
molecule of chitosan and takes place between $271{ }^{\circ} \mathrm{C}$ and $340{ }^{\circ} \mathrm{C}$, with a $T_{\max }$ at around $300{ }^{\circ} \mathrm{C}$. The weight loss assigned to this thermal degradation process generally becomes more important as functionalization degree decreases (Table 4). A characteristic temperature corresponding to the acetylated part, expected at around $400{ }^{\circ} \mathrm{C}$ in biopolymers with lower quantity of HMDI segments in their structures (CSAN-3 and CSAN-4), also appears in the thermograms. Moreover, a final decomposition stage with $T_{\max }$ at around $450{ }^{\circ} \mathrm{C}$ was detected, which can be assigned to excessively cross-linked chitosan fractions originating more rigid polyurethanes [63]. Thermal degradation of NCO-functionalized chitin polymers (Figure $2 \mathrm{~b}$ ) is rather similar to that discussed for chitosan samples. The loss of HMDI segments was found from $83{ }^{\circ} \mathrm{C}$ to $154{ }^{\circ} \mathrm{C}$, with maximum weight-loss rate temperatures between $119^{\circ} \mathrm{C}$ and $135^{\circ} \mathrm{C}$, whereas the other main thermal degradation event takes place at around $390{ }^{\circ} \mathrm{C}$, which corresponds to the loss of the acetylated units of the biopolymer. Finally, a third degradation stage occurs at $439-512{ }^{\circ} \mathrm{C}$, similarly to that found in chitosan samples.

Figure 2. Thermal degradation curves, under inert atmosphere, for NCO-functionalized chitosan (a) and chitin (b) samples and starting materials.

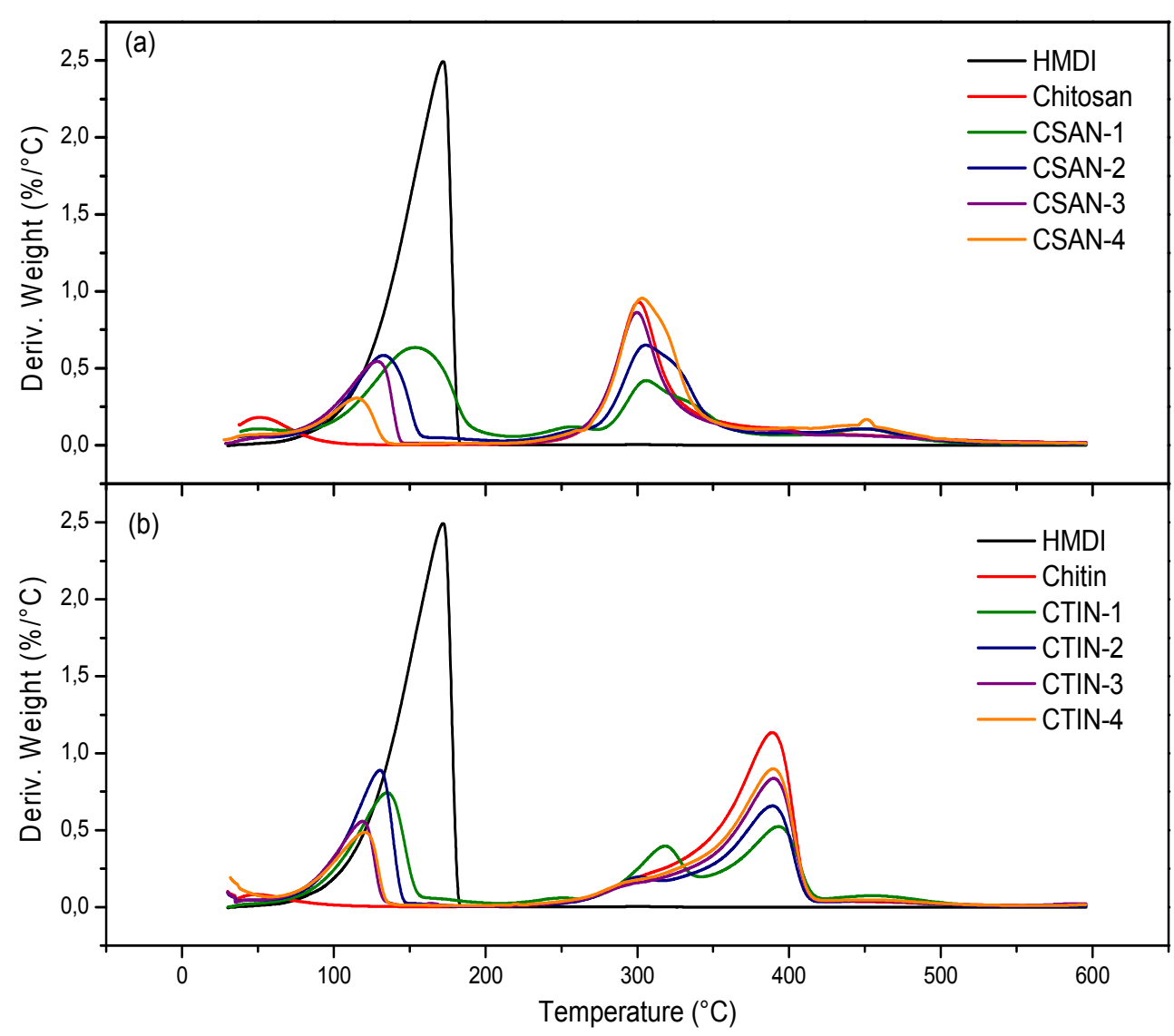


Table 4. TGA characteristic parameters for NCO-functionalized chitosan and chitin samples studied (main degradation stages in bold).

\begin{tabular}{cccccc}
\hline Sample & $\boldsymbol{T}_{\text {onset }}\left[{ }^{\circ} \mathbf{C}\right]$ & $\boldsymbol{T}_{\max }\left[{ }^{\circ} \mathbf{C}\right]$ & $\boldsymbol{T}_{\text {final }}\left[{ }^{\circ} \mathbf{C}\right]$ & $\Delta \mathbf{W}[\%]$ & Residue [\%] \\
\hline Chitosan & $\mathbf{2 8 1}$ & $\mathbf{3 0 1}$ & $\mathbf{4 7 2}$ & $\mathbf{5 7}$ & 34 \\
Chitin & $\mathbf{3 4 3}$ & $\mathbf{3 8 9}$ & $\mathbf{4 0 5}$ & $\mathbf{2 3}$ & 72 \\
HMDI & $\mathbf{1 3 7}$ & $\mathbf{1 7 2}$ & $\mathbf{1 7 8}$ & $\mathbf{9 9}$ & 1 \\
CSAN-1 & $\mathbf{1 2 2} / 240 / \mathbf{2 9 2} / 429$ & $\mathbf{1 5 3} / 258 / \mathbf{3 0 6} / 450$ & $\mathbf{1 7 9} / 266 / \mathbf{3 4 0} / 483$ & $\mathbf{4 4} / 6 / \mathbf{2 6} / 9$ & 11 \\
CSAN-2 & $\mathbf{1 0 7 / 2 8 3} / 431$ & $\mathbf{1 3 3} / \mathbf{3 0 6} / 448$ & $\mathbf{1 5 0} / \mathbf{3 3 5} / 486$ & $\mathbf{3 0} / \mathbf{4 3} / 9$ & 15 \\
CSAN-3 & $\mathbf{9 3} / \mathbf{2 7 1} / 391 / 416$ & $\mathbf{1 2 9} / \mathbf{3 0 0} / 395 / 443$ & $\mathbf{1 4 3} / \mathbf{3 2 4} / 410 / 520$ & $\mathbf{2 4} / \mathbf{4 1} / 3 / 7$ & 25 \\
CSAN-4 & $\mathbf{8 3} / \mathbf{2 7 4} / 388 / 445$ & $\mathbf{1 1 6 / 3 0 3} / 401 / 450$ & $\mathbf{1 3 3} / \mathbf{3 3 9} / 406 / 464$ & $\mathbf{1 4} / \mathbf{5 1} / 4 / 11$ & 20 \\
CTIN-1 & $\mathbf{9 3} / 224 / 283 / \mathbf{3 6 1} / 444$ & $\mathbf{1 3 5} / 251 / 318 / \mathbf{3 9 3} / 453$ & $\mathbf{1 5 4} / 260 / 334 / \mathbf{4 1 4} / 512$ & $\mathbf{3 4} / 2 / 17 / \mathbf{2 5} / 6$ & 16 \\
CTIN-2 & $\mathbf{1 0 6} / 275 / \mathbf{3 6 3} / 439$ & $\mathbf{1 3 0} / 302 / \mathbf{3 8 9} / 454$ & $\mathbf{1 4 0} / 309 / \mathbf{4 0 3} / 494$ & $\mathbf{3 4} / 10 / \mathbf{3 6} / 4$ & 16 \\
CTIN-3 & $\mathbf{8 3} / \mathbf{3 3 7} / 442$ & $\mathbf{1 1 9} / \mathbf{3 9 0} / 459$ & $\mathbf{1 3 2} / \mathbf{4 1 1} / 511$ & $\mathbf{2 2} / \mathbf{5 3} / 4$ & 21 \\
CTIN-4 & $\mathbf{8 3} / \mathbf{3 3 6} / 441$ & $\mathbf{1 2 1} / \mathbf{3 9 0} / 463$ & $\mathbf{1 3 4} / \mathbf{4 1 1} / 512$ & $\mathbf{2 2} / \mathbf{5 8} / 4$ & 16 \\
\hline
\end{tabular}

Thermal decomposition of NCO-functionalized chitosan- and chitin-based oleogels is shown in Figure 3. The thermal degradation of non-functionalized chitosan oleogel takes place from $278{ }^{\circ} \mathrm{C}$ to $482{ }^{\circ} \mathrm{C}$ and three different thermal events with $T_{\max }$ at $302{ }^{\circ} \mathrm{C}, 389{ }^{\circ} \mathrm{C}$ and $449{ }^{\circ} \mathrm{C}$ can be detected (Figure 3a, Table 5). The first one corresponds to chitosan degradation, the second one is mainly due to castor oil evaporation [30], the main oleogel component, and the last one refers to secondary products. For NCO-functionalized chitosan-based formulations (Figure 3a, Table 5), the thermal degradation occurs between $266{ }^{\circ} \mathrm{C}$ and $483{ }^{\circ} \mathrm{C}$, also in three different stages. The first one, at 302-307 ${ }^{\circ} \mathrm{C}$, is mainly noticed in those oleogels containing lower amounts of HMDI in their formulation (CSAN-3-30 and CSAN-4-30) and is attributed to the degradation of deacetylated chitin units. The second stage is again a consequence of castor oil evaporation. The temperature range for this event is expanded when the ratio of HMDI in the formulation increases (CSAN-1-30 and CSAN-2-30), which may be attributed to the urethane segments achieved during the reaction between functionalized chitosan and ricinoleic fatty chains. In the case of chitin-based oleogels (Figure $3 b$, Table 5), thermal decomposition always takes place in two clearly different stages. The main one can be observed between $324{ }^{\circ} \mathrm{C}$ and $410{ }^{\circ} \mathrm{C}$ and is a consequence of both thickener and castor oil degradations, which appear overlapped in one peak. The second minor event is observed at around $450{ }^{\circ} \mathrm{C}$ and refers to secondary products. Finally, it can be concluded that the disappearance of the first isocyanate-functionalized polymer degradation peak, due to HMDI decomposition, in the resulting oleogel confirms the chemical reaction promoted between modified polymers and the castor oil. 
Table 5. TGA characteristic parameters for non-functionalized and NCO-functionalized chitosan and chitin-based oleogels studied (main degradation stages in bold).

\begin{tabular}{cccccc}
\hline Sample & $\boldsymbol{T}_{\text {onset }}\left[{ }^{\circ} \mathbf{C}\right]$ & $\boldsymbol{T}_{\max }\left[{ }^{\circ} \mathbf{C}\right]$ & $\left.\boldsymbol{T}_{\text {final }}{ }^{\circ} \mathbf{C}\right]$ & Residue $[\%]$ & $\Delta \mathbf{W}[\mathbf{\%}]$ \\
\hline CSAN-30 & $278 / \mathbf{3 5 7}$ & $302 / \mathbf{3 8 9} / 449$ & $319 / \mathbf{4 8 2}$ & 4 & $19 / 77$ \\
CSAN-1-30 & $\mathbf{2 9 7}$ & $344 / \mathbf{3 8 6} / 452$ & $\mathbf{4 8 1}$ & 8 & $\mathbf{9 2}$ \\
CSAN-2-30 & $\mathbf{3 0 1}$ & $339 / \mathbf{3 8 9} / 451$ & $\mathbf{4 8 1}$ & 10 & $\mathbf{9 0}$ \\
CSAN-3-30 & $276 / \mathbf{3 5 3} / 433$ & $307 / \mathbf{3 8 6} / 447$ & $321 / \mathbf{4 1 2} / 483$ & 6 & $17 / \mathbf{6 6} / 11$ \\
CSAN-4-30 & $266 / 355$ & $302 / \mathbf{3 8 7} / 443$ & $319 / 481$ & 8 & $26 / \mathbf{6 6}$ \\
CTIN-30 & $\mathbf{3 4 0} / 441$ & $\mathbf{3 8 1} / 459$ & $\mathbf{4 0 9} / 495$ & 11 & $\mathbf{8 0} / 9$ \\
CTIN-1-30 & $\mathbf{3 2 4} / 439$ & $\mathbf{3 7 9} / 446$ & $\mathbf{4 1 0} / 482$ & 3 & $\mathbf{8 3} / 14$ \\
CTIN-2-30 & $\mathbf{3 2 7} / 444$ & $\mathbf{3 7 6} / 450$ & $\mathbf{4 0 5} / 485$ & 5 & $\mathbf{8 2} / 13$ \\
CTIN-3-30 & $\mathbf{3 2 6} / 443$ & $\mathbf{3 7 4} / 449$ & $\mathbf{4 0 6} / 462$ & 6 & $\mathbf{8 3} / 11$ \\
CTIN-4-30 & $\mathbf{3 2 8} / 425$ & $\mathbf{3 7 3} / 449$ & $\mathbf{4 0 4} / 486$ & 4 & $\mathbf{8 6} / 10$ \\
\hline
\end{tabular}

Figure 3. Thermal degradation curves, under inert atmosphere, for NCO-functionalized chitosan-(a) and chitin-(b) based oleogels.

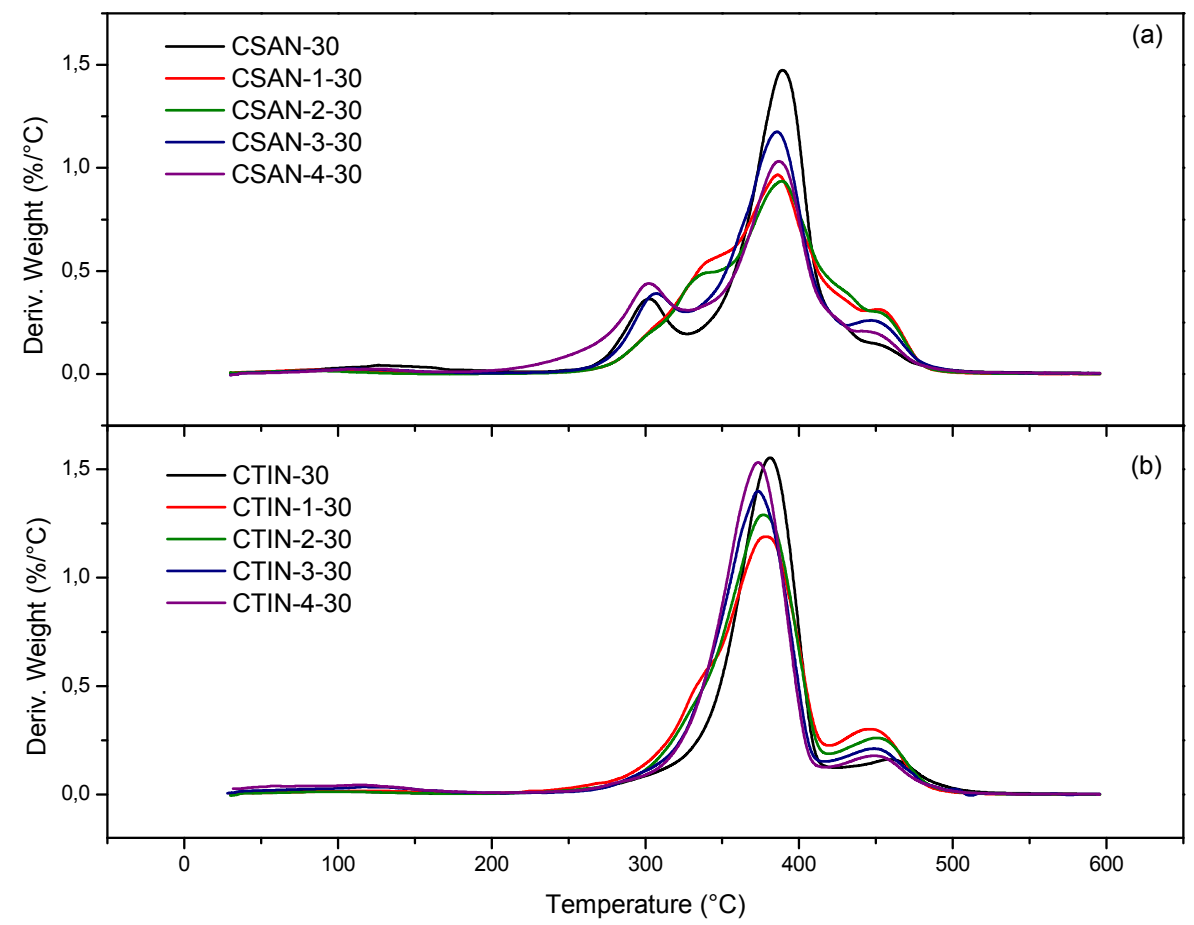

\subsection{Rheology of Chitosan- and Chitin-Based Oleogels}

Figure 4 shows the SAOS response, inside the linear viscoelastic range, of NCO-functionalized and non-functionalized chitosan and chitin-based oleogels, at $30 \% \mathrm{w} / \mathrm{w}$ concentration, as a function of the functionalization degree. In most cases, NCO-functionalized chitosan-based oleogels exhibit values of the storage modulus, $\mathrm{G}^{\prime}$, significantly higher than those found for the loss modulus, $\mathrm{G}^{\prime \prime}$, in the whole frequency range studied (Figure 4a). This evolution, the so-called "plateau region", where G' slightly increases with frequency and $\mathrm{G}^{\prime \prime}$ displays a minimum, is typically found in highly entangled polymeric systems [64] and standard lubricating greases [65]. Only when using the lowest amount of HMDI (CSAN-4-30), the plateau region appears to be not well developed, indicating the consecution of a 
weaker gel. The plateau region is also exhibited by some of the NCO-functionalized chitin-based oleogels such as CTIN-3-30 and CTIN-4-30 (Figure 4b). However, interestingly, a crossover between both viscoelastic functions was found at high frequency for the highly-functionalized chitin-based oleogels (CTIN-1-30 and CTIN-2-30). In these cases, this behaviour must be attributed to a higher degree of cross linking achieved during oleogel formation, resulting in a rheological response closer to the transition region of the mechanical spectrum. In general, as can be deduced from Figure $4,-\mathrm{NCO}$ content in the functionalized biopolymers largely influences the values of the linear viscoelastic functions of resulting oleogels. In the case of chitosan, a high degree of functionalization (CSAN-1-30 and CSAN-2-30) increases the values of the SAOS functions with respect to those shown by the non-functionalized chitosan oleogel (CSAN-30), as a consequence of an extensive crosslinking. However, the values of the SAOS functions decrease when reducing the amount of HMDI incorporated to the biopolymer, yielding similar (CSAN-3-30) or lower (CSAN-4-30) $\mathrm{G}^{\prime}$ and $\mathrm{G}^{\prime \prime}$ values than those exhibited by the non-functionalized chitosan-based oleogel (CSAN-30).

Figure 4. Frequency dependence of the storage, $G^{\prime}$, and loss, $G^{\prime \prime}$, moduli for (a) NCO-functionalized chitosan-based oleogels (aged for 4 months) and (b) chitin-based oleogels (aged for 1 month), as a function of functionalization degree.

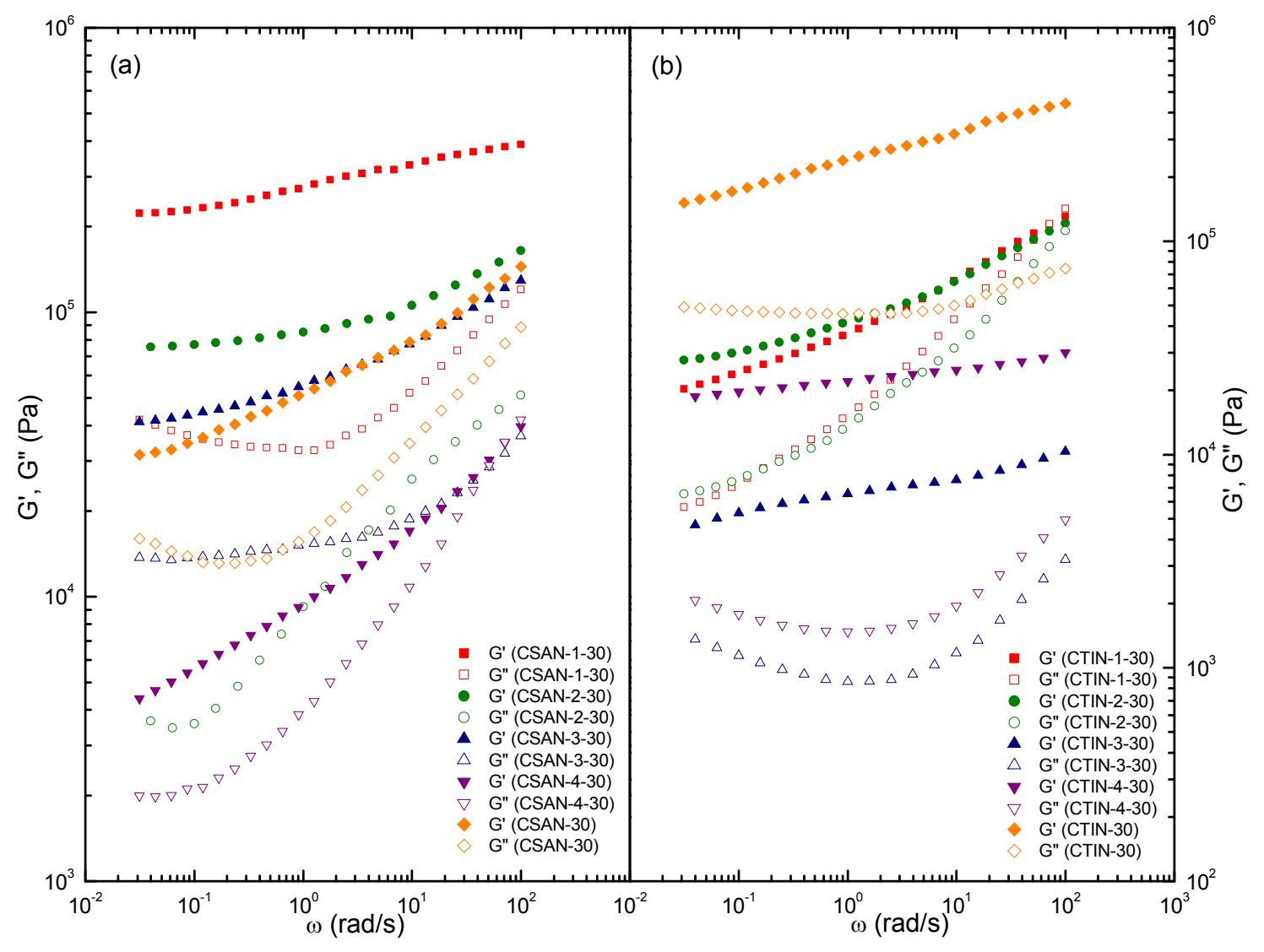

On the contrary, for chitin-based oleogels, the most noticeable effect of HMDI segments is that they always produce lower values of the viscoelastic moduli than those shown by the non-functionalized chitin-based oleogel (CTIN-30). Apart from this, oleogels with high amounts of HMDI in their formulation (CTIN-1-30 and CTIN-2-30) exhibited the highest values of the linear viscoelastic 
functions but, contrary to the expected, CTIN-3-30 showed lower values than CTIN-4-30. All these effects that functionalization degree exerts on the rheological response of chitin- and chitosan-based oleogels can be more noticeably summarized in Figure 5, where the values of $\mathrm{G}^{\prime}$ and the loss tangent $\left(\tan \delta=\mathrm{G}^{\prime \prime} / \mathrm{G}^{\prime}\right)$, at $1 \mathrm{rad} \cdot \mathrm{s}^{-1}$, are plotted versus the HMDI proportion applied in the functionalization of biopolymers. As can be observed, the insertion of low amounts of HMDI in both chitin and chitosan produces a drastic reduction in the values of SAOS functions, i.e., $\mathrm{G}_{1}^{\prime}\left(\mathrm{G}^{\prime}\right.$ at $\left.1 \mathrm{rad} \cdot \mathrm{s}^{-1}\right)$ especially for chitosan-based oleogels. However, above a critical threshold, SAOS functions increase with the functionalization degree. Interestingly, this increase in both $\mathrm{G}^{\prime}$ and $\mathrm{G}^{\prime \prime}$ is associated to a lower relative elasticity in the case of chitin-based oleogels and higher in the case of chitosan-based ones, as can be seen in the $\tan \delta v s$. HMDI proportion plot (Figure 5). As mentioned above, only in the case of chitosan-based oleogels a high degree of functionalization makes possible to reach values of the viscoelastic moduli higher than those displayed by the non-functionalized biopolymer-based oleogel. However, as previously reported [27,42], it must be remarked that these physically stabilized gels present some limitations regarding their physical and mechanical stability, which can be overcome by mean of functionalization.

Figure 5. HMDI proportion dependence of the storage modulus, $\mathrm{G}_{1}^{\prime}\left(\mathrm{G}^{\prime}\right.$ at $\left.1 \mathrm{rad} \cdot \mathrm{s}^{-1}\right)$, and the loss tangent, $\tan \delta$, at $1 \mathrm{rad} \cdot \mathrm{s}^{-1}$, for NCO-functionalized chitosan- and chitin-based oleogels.

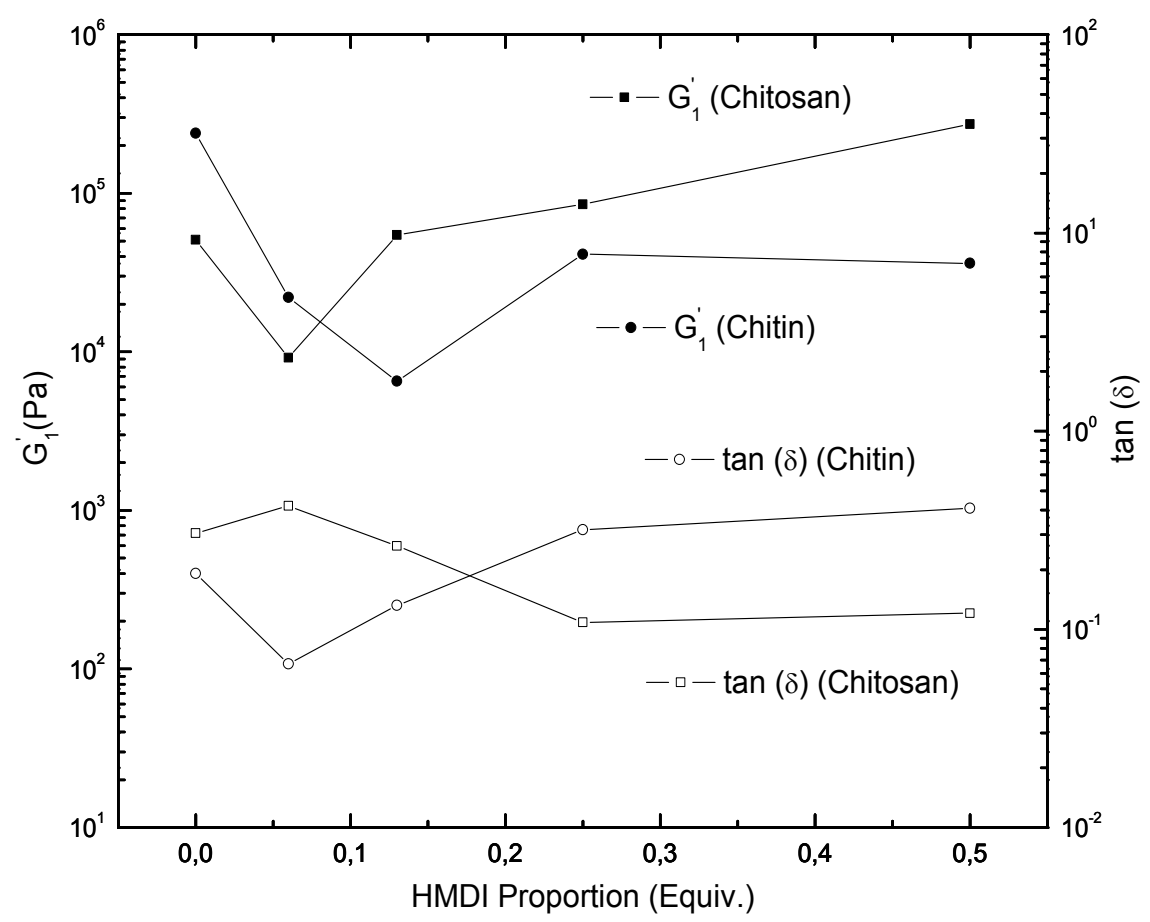

As extensively investigated [66-68], typical $\mathrm{G}^{\prime}$ values in conventional lubricating greases of NLGI (National Lubricating Grease Institute) grade 1-2 range from $10^{4}-10^{5} \mathrm{~Pa}$, around one order of magnitude higher than $\mathrm{G}^{\prime \prime}$ values, depending on compositions and processing conditions. This particular rheological response has been obtained for several of the NCO-functionalized chitosan- and chitin-based oleogels studied, emphasizing that the degree of NCO-functionalization can be used as a chemical tool to modulate the rheological behaviour and consistency of these oleogels as desired. 
Rheological data collected in Figure 4a,b correspond to oleogels 1 and 4 months aged, respectively. As previously reported for NCO-functionalized methyl cellulose-based oleogels [42], these chemical gels require a certain ageing time to obtain stable rheological properties. Figure 6 shows the evolution of the linear viscoelastic functions with frequency, as a function of ageing time, for selected chitosan(CSAN-3-25) and chitin- (CTIN-1-30) based oleogels. In most of the cases studied, the values of SAOS functions significantly increase during the first seven days of ageing and then slightly increase or remain almost constant after several months of ageing. These results can be explained on the basis of the slow reactivity of residual -NCO functional groups in a high viscous medium [69]. However, interestingly, the loss tangent was almost unaffected (data not shown).

Figure 6. Frequency dependence of the storage, $\mathrm{G}^{\prime}$, and loss, G", moduli for selected NCO-functionalized chitosan- (CSAN-3-25) and chitin- (CTIN-1-30) based oleogels, as a function of ageing time.

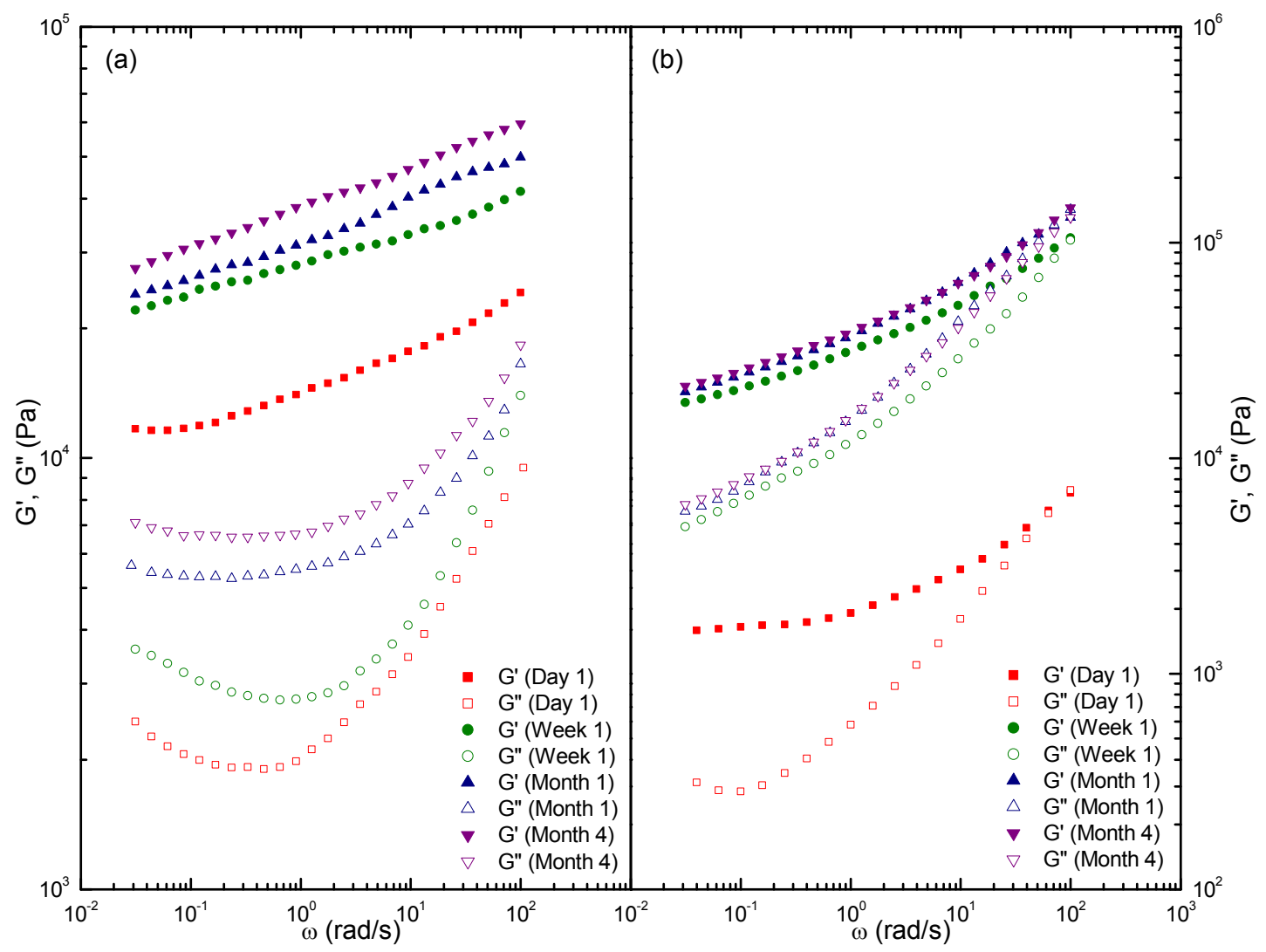

Finally, as typically performed in conventional lubricating greases, the rheological response can be modified by changing the thickener concentration, in this case the functionalized biopolymers used as gelling agents. Figure 7 shows the mechanical spectra of functionalized chitosan- and chitin-based oleogels, containing different concentrations of the same biopolymer in their respective formulations. As can be observed, the values of the viscoelastic moduli increase with the concentration of both functionalized biopolymers. In the case of chitosan-based oleogels (Figure 7a), G' exhibits values significantly higher than those found for $\mathrm{G}^{\prime \prime}$, i.e., the plateau region, for concentrations ranging from 25\%-35\% w/w (CSAN-3-25, CSAN-3-30 and CSAN-3-35). Nevertheless, a liquid-like weak gel was obtained with a $15 \%$ biopolymer concentration (CSAN-3-15). For chitin-based oleogels (Figure 7b), 
almost three decades of both SAOS functions can be covered by modifying the thickener concentration between $15 \%$ and $30 \% \mathrm{w} / \mathrm{w}$. In all cases, $\mathrm{G}^{\prime}$ is higher than $\mathrm{G}^{\prime \prime}$ at low frequencies. However, a crossover between both linear viscoelastic functions was always observed, which is shifted to lower frequencies as biopolymer concentration decreases.

Figure 7. Frequency dependence of the storage, $\mathrm{G}^{\prime}$, and loss, G", moduli for NCO-functionalized chitosan-(a) and chitin-(b) based oleogels, as a function of biopolymer concentration.

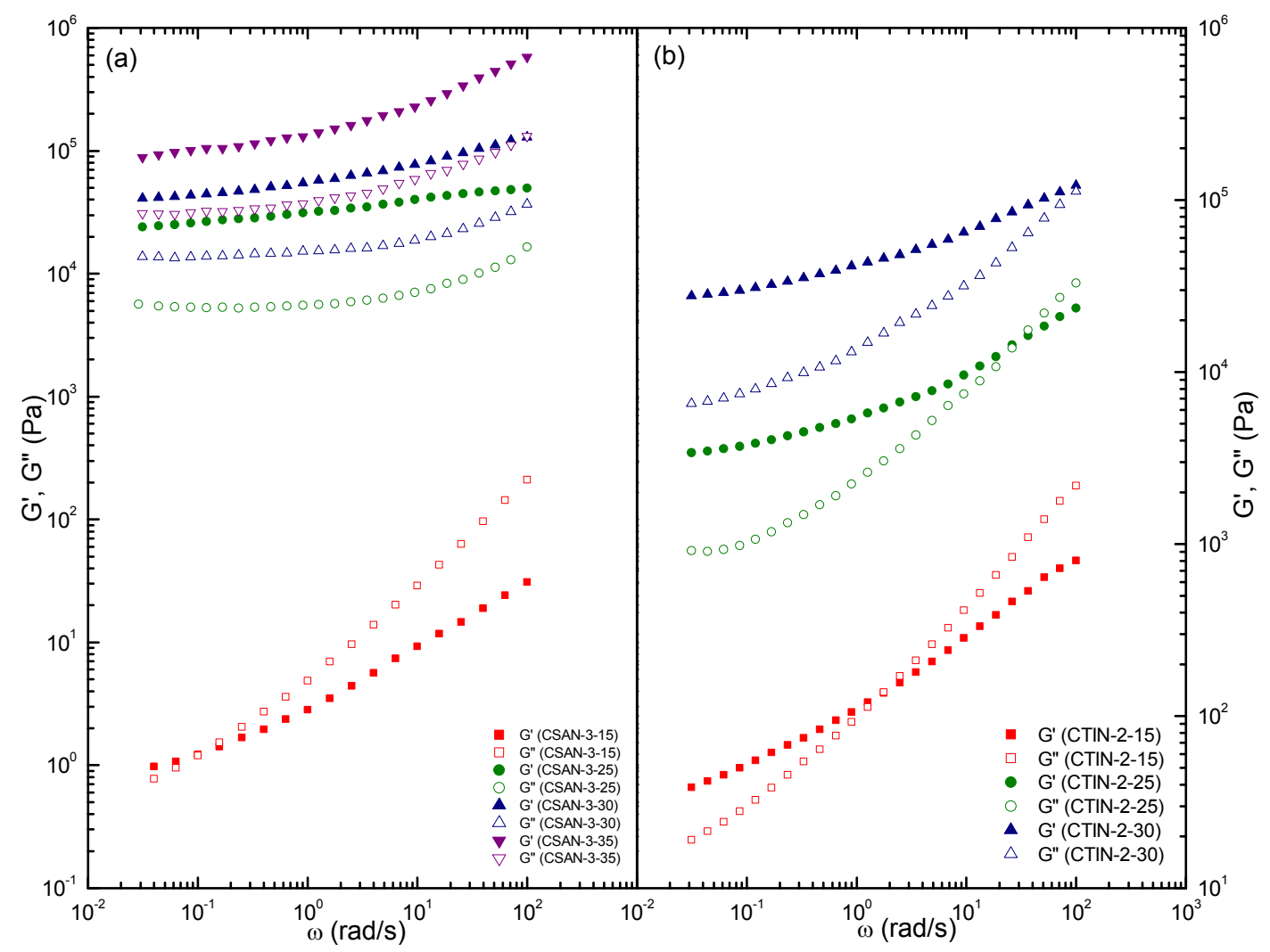

\section{Conclusions}

A systematic and controlled functionalization protocol for chitosan and chitin with HMDI resulted in several biopolymers functionalized in different degrees. The dispersion of these biopolymers in castor oil promoted the reaction between free - NCO segments of the biopolymer and $-\mathrm{OH}$ groups of the ricinoleic fatty acid chain thus providing different chemical oleogels with specific characteristics.

The resulting oleogels presented suitable thermal resistance despite the fact that the inclusion of HMDI segments in the polymer structure reduces their thermal stabilities. Oleogels thermal degradation basically takes place in one main stage which comprises the thermal decomposition of both the castor oil and the thickening agent.

Typical rheological behaviour found in conventional lubricating greases was achieved in different formulations studied, providing a cross-linked viscoelastic gel with a well-developed plateau region in the evolution of SAOS functions with frequency. The insertion of low amounts of HMDI in both chitin and chitosan produces a drastic reduction in the values of SAOS functions but, above a critical 
threshold, viscoelastic functions increase with the functionalization degree. Therefore, isocyanate functionalization appears as a chemical tool to modulate the rheological response.

The rheological properties of functionalized chitosan- and chitin-based oleogels evolve during several months of ageing, but mainly during the first week, due to the progress of the reaction between $-\mathrm{NCO}$ functional groups and castor oil -OH groups. Oleogel linear viscoelastic response is also highly dependent on the concentration of NCO-functionalized biopolymers.

Several NCO-functionalized chitosan- and chitin-based oleogel formulations present suitable thermal resistance and rheological characteristics to be proposed as bio-based alternatives to traditional lubricating greases.

\section{Acknowledgments}

This work is part of two research projects (CTQ2010-15338 and TEP-1499) sponsored by MINECO-FEDER (70\% European cofunding rate) and Junta de Andalucía programmes, respectively. One of the authors (Rocío Gallego) has received a Ph.D. Research Grant from DIGICyT (MINECO). The authors gratefully acknowledge the financial support.

\section{Author Contributions}

All the authors have contributed equally to this work.

\section{Conflicts of Interest}

The authors declare no conflict of interest.

\section{References}

1. Dutty, N.; Blonk, H.C.G.; Beindorff, C.M.; Cazade, M.; Bot, A.; Duchateau, G.S.M.J.E. Organogel-based emulsion systems, micro-structural features and impact on in vitro digestion. J. Am. Oil Chem. Soc. 2009, 86, 733-741.

2. Wang, K.K.; Jia, Q.A.; Han, F.; Liu, H.Z.; Li, S.M. Self-assembled L-alanine derivative organogel as in situ drug delivery implant: Characterization, biodegradability, and biocompatibility. Drug Dev. Ind. Pharm. 2010, 36, 1511-1521.

3. Lupi, F.R.; Gabriele, D.; Baldino, N.; Mijovic, P.; Parisi, O.I.; Puoci, F. Olive oil/policosanol organogels for nutraceutical and drug delivery purposes. Food Funct. 2013, 4, 1512-1520.

4. Satapathy, D.; Biswas, D.; Behera, B.; Sagiri, S.S.; Pal, K.; Pramanik, K. Sunflower-oil-based lecithin organogels as matrices for controlled drug delivery. J. Appl. Polym. Sci. 2013, 129, 585-594.

5. Terech, P.; Weiss, R.G. Low-mass luminescent organogels. In Surface Characterization Methods: Principles, Techniques and Applications, Milling, A.J., Ed.; Marcel Dekker: New York, NY, USA, 1999; pp. 285-344.

6. Bag, B.G.; Dinda, S.K.; Dey, P.P.; Mallia, V.A.; Weiss, R.G. Self-assembly of esters of arjunolic acid into fibrous networks and the properties of their organogels. Langmuir 2009, 25, 8663-8671. 
7. Banerjee, S.; Das, R.K.; Terech, P.; de Geyer, A.; Aymonier, C.; Loppinet-Serani, A.; Raffy, G.; Maitra, U.; del Guerzo, A.; Desvergne, J.P. Hybrid organogels and aerogels from co-assembly of structurally different low molecular weight gelators. J. Mater. Chem. C 2013, 1, 3305-3316.

8. Bot, A.; Veldhuizen, Y.S.J.; den Adel, R.; Roijers, E.C. Non-TAG structuring of edible oils and emulsions. Food Hydrocoll. 2009, 23, 1184-1189.

9. Lupi, F.R.; Gabriele, D.; Baldino, N.; Seta, L.; de Cindio, B.; de Rose, C. Stabilization of meat suspensions by organogelation: A rheological approach. Eur. J. Lipid Sci. Technol. 2012, 114, 1381-1389.

10. Carretti, E.; Dei, L.; Weiss, R.G.; Baglioni, P. A new class of gels for the conservation of painted surfaces. J. Cultural Herit. 2008, 9, 386-393.

11. Carretti, E.; Bonini, M.; Dei, L.; Berrie, B.H.; Angelova, L.V.; Baglioni, P.; Weiss, R.G. New frontiers in materials sciences for art conservation: responsive gels and beyond. Acc. Chem. Res. 2010, 43, 751-760.

12. Terech, P.; Weiss, R.G. Low molecular mass gelators of organic liquids and the properties of their gels. Chem. Rev. 1997, 97, 3133-3160.

13. George, M.; Funkhouser, G.P.; Weiss, R.G. Organogels with complexes of ions and phosphorus-containing amphiphiles as gelators. Spontaneous gelation by in situ complexation. Langmuir 2008, 24, 3537-3544.

14. Mallia, V.A.; George, M.; Blair, D.L.; Weiss, R.G. Robust organogels from nitrogen-containing derivatives of $(R)$-12-Hydroxystearic acid as gelators: Comparisons with gels from Stearic acid derivatives. Langmuir 2009, 25, 8615-8625.

15. Sawalha, H.; Venema, P.; Bot, A.; Floter, E.; van der Linden, E. The influence of concentration and temperature on the formation of gamma-oryzanol + I-2-sitosterol tubules in edible oil organogels. Food Biophys. 2011, 6, 20-25.

16. D’Anna, F.; Vitale, P.; Marullo, S.; Noto, R. Geminal imidazolium salts: A new class of gelators. Langmuir 2012, 28, 10849-10859.

17. Toro-Vazquez, J.F.; Morales-Rueda, J.; Torres-Martinez, A.; Charo-Alonso, M.A.; Mallia, V.A.; Weiss, R.G. Cooling rate effects on the microstructure, solid content and rheological properties of organogels of amides derived from Stearic and $(R)$-12-hydroxystearic acid in vegetable oil. Langmuir 2013, 29, 7642-7654.

18. Morales-Rueda, J.A.; Dibildox-Alvarado, E.; Charo-Alonso, M.A.; Toro-Vázquez, J.F. Rheological properties of Candelilla wax and dotriacontane organogels measured with a true-gap system. J. Am. Oil Chem. Soc. 2009, 86, 765-772.

19. Toro-Vazquez, J.F.; Morales-Rueda, J.; Mallia, V.A.; Weiss, R.G. Relationship between molecular structure and thermo-mechanical properties of Candelilla wax and amides derived from (R)-12-hydroxystearic acid as gelators of safflower oil. Food Biophys. 2010, 5, 193-202.

20. Laredo, T.; Barbut, S.; Marangoni, A.G. Molecular interactions of polymer oleogelation. Soft Matter. 2011, 7, 2734-2743.

21. Co, E.D.; Marangoni, A.G. Organogels: An alternative edible oil-structuring method. J. Am. Oil Chem. Soc. 2012, 89, 749-780.

22. Zetzl, A.K.; Marangoni, A.G.; Barbut, S. Mechanical properties of ethylcellulose oleogels and their potential for saturated fat reduction in frankfurters. Food Funct. 2012, 3, 327-337. 
23. Alvarez-Mitre, F.M.; Toro-Vazquez, J.M.; Moscosa-Santillan, M. Shear rate and cooling modeling for the study of Candelilla wax organogels' rheological properties. J. Food Eng. 2013, 119, 611-618.

24. Patel, A.R.; Schatteman, D.; Lesaffer, A.; Dewettinck, K. A foam-templated approach for fabricating organogels using a water-soluble polymer. RSC Adv. 2013, 3, 22900-22903.

25. Patel, A.R.; Schatteman, D.; de Vos, W.H.; Lesaffer, A.; Dewettinck, K. Preparation and rheological characterization of shellac oleogels and oleogel-based emulsions. J. Colloid Interface Sci. 2013, 411, 114-121.

26. Gravelle, A.J.; Barbut, S.; Marangoni, A.G. Fractionation of ethylcellulose oleogels during setting. Food Funct. 2013, 4, 153-161.

27. Sánchez, R.; Franco, J.M.; Delgado, M.A.; Valencia, C.; Gallegos, C. Development of new green lubricating grease formulations based on cellulosic derivatives and castor oil. Green Chem. 2009, 11, 686-693.

28. Sánchez, R.; Franco, J.M.; Delgado, M.A.; Valencia, C.; Gallegos, C. Thermal and mechanical characterization of cellulosic derivatives-based oleogels potentially applicable as biolubricating greases: Influence of ethyl cellulose molecular weight. Carbohydr. Polym. 2011, 83, 151-158.

29. Sánchez, R.; Franco, J.M.; Delgado, M.A.; Valencia, C.; Gallegos, C. Rheological and mechanical properties of oleogels based on castor oil and cellulosic derivatives potentially applicable as bio-lubricating greases: Influence of cellulosic derivatives concentration ratio. J. Ind. Eng. Chem. 2011, 17, 705-711.

30. Núñez, N.; Martín-Alfonso, J.E.; Eugenio, M.E.; Valencia, C.; Díaz, M.J.; Franco, J.M. Preparation and characterization of gel-like dispersions based on cellulosic pulps and castor oil for lubricant applications. Ind. Eng. Chem. Res. 2011, 50, 5618-5627.

31. Núñez, N.; Martín-Alfonso, J.E.; Eugenio, M.E.; Valencia, C.; Díaz, M.J.; Franco, J.M. Influence of eucalyptus globulus Kraft pulping severity on the rheological properties of gel-like cellulose pulp dispersions in castor oil. Ind. Eng. Chem. Res. 2012, 51, 9777-9782.

32. Núñez, N.; Martín-Alfonso, J.E.; Valencia, C.; Sánchez, M.C.; Franco, J.M. Rheology of new green lubricating grease formulations containing cellulose pulp and its methylated derivative as thickener agents. Ind. Crop. Prod. 2012, 37, 500-507.

33. Lea, C.W. European development of lubricant derived from renewable resources. Ind. Lubr. Tribol. 2002, 54, 268-274.

34. Wilson, B. Lubricants and functional fluids from renewable sources. Ind. Lubr. Tribol. 1998, 50, 6-15.

35. Gryglewicz, S.; Muszynski, M.; Nowicki, J. Enzymatic synthesis of rapeseed oil-based lubricants. Ind. Crop. Prod. 2013, 45, 25-29.

36. Hashem, A.I.; AbouElmagd, W.S.I.; Salem, A.E.; El-Kasaby, M.; El-Nahas, A.M. Conversion of some vegetable oils into synthetic lubricants. Energy Source A 2013, 35, 397-400.

37. Zulkifli, N.W.M.; Kalam, M.A.; Masjuki, H.H.; Shahabuddin, M.; Ynus, R. Wear characteristics of a palm oil-based TMP (trimethylolpropane) ester as an engine lubricant. Energy 2013, 54, 167-173.

38. Salih, N.; Salimon, J.; Jantan, F.N. Synthesis and characterization of palm kernel oil based trimethylolpropane ester. Asian J. Chem. 2013, 25, 9751-9754. 
39. García-Zapateiro, L.A.; Franco, J.M.; Valencia, C.; Delgado, M.A.; Gallegos, C. Viscous, thermal and tribological characterization of oleic and ricinoleic acids-derived estolides and their blends with vegetable oils. J. Ind. Eng. Chem. 2013, 19, 1289-1298.

40. García-Zapateiro, L.A.; Franco, J.M.; Valencia, C.; Delgado, M.A.; Gallegos, C.; Ruiz-Mendez, M.V. Chemical, thermal and viscous characterization of high-oleic sunflower and olive pomace acid oils and derived estolides. Grasas y Aceites 2013, 64, 497-508.

41. Gallego, R.; Arteaga, J.F.; Valencia, C.; Franco, J.M. Chemical modification of methyl cellulose with HMDI to modulate the thickening properties in castor oil. Cellulose 2013, 20, 495-507.

42. Gallego, R.; Arteaga, J.F.; Valencia, C.; Franco, J.M. Rheology and thermal degradation of isocyanate-functionalized methyl cellulose-based oleogels. Carbohydr. Polym. 2013, 98, 152-160.

43. Gallego, R.; Arteaga, J.F.; Valencia, C.; Franco, J.M. Isocyanate-functionalized chitin and chitosan as gelling agents of castor oil. Molecules 2013, 18, 6532-6549.

44. Rinaudo, M. Chitin and chitosan: properties and applications. Prog. Polym. Sci. 2006, 31, 603-632.

45. Alves, N.M.; Mano, J.F. Chitosan derivatives obtained by chemical modifications for biomedical and environmental applications. Int. J. Biol. Macromol. 2008, 43, 401-414.

46. Dias, A.M.A.; Cortez, A.R.; Barsan, M.M.; Santos, J.B.; Brett, C.M.A.; de Sousa, H.C. Development of greener multi-responsive chitosan biomaterials doped with biocompatible ammonium ionic liquids. ACS Sustain. Chem. Eng. 2013, 1, 1480-1492.

47. Kumar, R.; Muzzarelli, M.N.V.; Muzzarelli, R.A.A.; Sashiwa, C.H.; Domb, A.J. Chitosan chemistry and pharmaceutical perspectives. Chem. Rev. 2004, 104, 6017-6084.

48. Skjak-Braek, G.; Anthonsen, T.; Sandford, P.A. Chitin and Chitosan-Sources, Chemistry, Biochemistry, Physical Properties and Applications; Elsevier: London, UK, 1989; pp. 51-70.

49. Muzzarelli, R.A.A. Genepin-crosslinked chitosan hydrogels as biomedical and pharmaceutical aids. Carbohydr. Polym. 2009, 77, 1-9.

50. Bersch, P.C.; Nies, B.; Liebendorfer, A. Evaluation of the biological properties of different wound dressing materials. J. Mater. Sci. Mater. M 1995, 6, 231-240.

51. Kurita, K.; Kojima, T.; Munakata, T.; Akao, H.; Mori, T.; Nishiyama, Y. Preparation of non-natural branched chitin and chitosan. Chem. Lett. 1998, 27, 317-318.

52. Sashiwa, H.; Shigemasa, Y. Chemical modification of chitin and chitosan 2: Preparation and water soluble property of $N$-acylated or $N$-alkylated partially deacetylated chitins. Carbohydr. Polym. 1999, 39, 127-138.

53. Heras, A.; Rodriguez, N.M.; Ramos, V.M. N-methylene phosphonic chitosan: A novel soluble derivative. Carbohydr. Polym. 2001, 44, 1-8.

54. Rinaudo, M. New way to crosslink chitosan in aqueous solution. Eur. Polym. J. 2010, 46, 1537-1544.

55. Prabaharan, M.; Mano, J.F. Chitosan derivatives bearing cyclodextrin cavities as novel adsorbent matrices. Carbohydr. Polym. 2006, 63, 153-166.

56. Chiu, S.; Chung, T.; Giridhar, R.; Wu, W. Immobilization of $\beta$-cyclodextrin in chitosan beads for separation of cholesterol from egg yolk. Food Res. Int. 2004, 37, 217-223.

57. Chen, S.; Tsao, C.; Chang, C.; Wu, Y.; Liu, Z.; Lin, C.; Wang, C.; Hsieh, K. Synthesis and characterization of thermal-responsive chitin-based polyurethane copolymer as a smart material. Carbohydr. Polym. 2012, 88, 1483-1487. 
58. Ziaa, K.M.; Bhattia, I.A.; Barikanib, M.; Zuberc, M.; Sheikh. M.A. XRD studies of chitin-based polyurethane elastomers. Int. J. Biol. Macromol. 2008, 43, 136-141.

59. Baxter, A.; Dillon, M.; Taylor, K.D.A.; Roberts, G.A.F. An Improved Method for IR Determination of the Degree of N-Acetylationof Chitosan. Int. J. Biol. Macromol. 1992, 14, 166-169.

60. Poirier, M.; Charlet, G. Chitin fractionation and characterization in $N, N$-dimethylacetamide/lithium chloride solvent system. Carbohydr. Polym. 2002, 50, 363-370

61. Takahashi, T.; Imai, M.; Suzuki, I. High-potential molecular properties of chitosan and reaction conditions for removing p-quinone from the aqueous phase. Biochem. Eng. J. 2005, 25, 7-13.

62. Paulino, A.T.; Simionato, J.I.; Garcia, J.C.; Nozaki, J. Characterization of chitosan and chitin produced from silkworm chrysalides. Carbohydr. Polym. 2006, 64, 98-103.

63. Mythili, C.V.; Malar Retna, A.; Gopalakrishnan, S. Synthesis, mechanical, thermal and chemical properties of polyurethanes based on cardanol. Bull. Mater. Sci. 2004, 27, 235-241.

64. Gupta, R.K. Polymer and Composite Rheology, 2nd ed.; Marcel Dekker: New York, NY, USA, 2000.

65. Sánchez, M.C.; Franco, J.M.; Valencia, C.; Gallegos, C.; Urquiola, F.; Urchegui, R. Atomic force microscopy and thermo-rheological characterization of lubricating greases. Tribol. Lett. 2011, 41, 463-470.

66. Martín-Alfonso, J.E.; Valencia, C.; Sánchez, M.C.; Franco, J.M.; Gallegos, C. Rheological modification of lubricating greases with recycled polymers from different plastics waste. Ind. Eng. Chem. Res. 2009, 48, 4136-4144.

67. Martin-Alfonso, J.E.; Valencia, C.; Sanchez, M.C.; Franco, J.M.; Gallegos, C. Development of new lubricating grease formulations using recycled LDPE as rheology modifier additive. Eur. Polym. J. 2007, 43, 139-149.

68. Franco, J.M.; Delgado, M.A.; Valencia, C.; Sánchez, M.C.; Gallegos, C. Mixing rheometry for studying the manufactured of lubricating greases. Chem. Eng. Sci. 2005, 60, 2409-2418.

69. Moreno, G.; Valencia, C.; Franco, J.M.; Gallegos, C.; Diogo, A.; Bordado, J.C.M. Influence of molecular weight and free $\mathrm{NCO}$ content on the rheological properties of lithium lubricating greases modified with NCO-terminated prepolymers. Eur. Polym. J. 2008, 44, 2262-2274.

(C) 2014 by the authors; licensee MDPI, Basel, Switzerland. This article is an open access article distributed under the terms and conditions of the Creative Commons Attribution license (http://creativecommons.org/licenses/by/3.0/). 